\title{
UnCANNY VALLEY PROJECT, UNA INTERVENCIÓN CIBERFEMINISTA EN la X Bienal Centroamericana
}

\author{
Uncanny Valley Project, a Cyberfeminist Intervention in the X Central American Biennial
}

\author{
Susan Campos-Fonseca
}

Resumen: En el presente testimonio se comparte una reflexión sobre la intervención ciberfeminista Uncanny Valley Project en la X Sonora de la Bienal Centroamericana (2016). Se analiza la intervención desde una "proximidad crítica, corporal y feminista", según propone Tamara Díaz y bajo la hipótesis del uncanny valley de Masahiro Mori, conel ánimo de exponer cómoésta actúa sobre un presente en crisis, junto con la música electrónica en tanto vía para construir cuerpos de invención e innovación. Además, se explora laperformatividady la estética robótica practicadas por humanos dedicados a la música electrónica. El resultado permite identificar el fenómeno dentro de la música electrónica costarricense, darle visibilidad en el contexto centroamericano y subrayar el aporte de las creadoras.

Palabras clave: investigación social, investigación artística, música electrónica centroamericana, ciberfeminismo.

Abstract: This article/testimony discusses a cyber-feminist intervention entitled Uncanny Valley Project, part of the $10^{\text {th }}$ Biennial CentroAmerican celebration (2016). This intervention is analyzed from a critical close-up perspective of the "corporal" and "feminist", categories proposed by Tamara Diaz; the hypothesis of Uncanny Valley as put forth by Masahiro Mori will also be utilized. The intent is to explore how these ideas/interventions together with electronic music, function in the current period of crisis as a means to construct bodies that invent and innovate; concepts of performance and robotic esthetics as practiced by people interested in electronic music will be considered. As a result of this investigation, we hope to position this intervention within the Costa Rican electronic music movement, shedding light on the larger Central American context, and highlighting the contributions of its creators.

Keywords: social research, artistic research, Centro American electronic music, cyber-feminism.

Susan Campos Fonseca. Doctora en Música por la Universidad Autónoma de Madrid, España, y doctora en Estudios de la Sociedad y la Cultura por la Universidad de Costa Rica. Profesora-investigadora en la Universidad de Costa Rica. Temas de especialización: filosofía de la cultura y de la creación sonora. Correo electrónico: susanconductor@gmail.com, SUSAN. CAMPOS_F@ucr.ac.cr.
Enviado a dictamen: 30 de agosto de 2016

Aprobación: 16 de marzo de 2017.

Revisiones: 1 . 


\section{Pensar desde una proximidad crítica}

E n su introducción a Crítica próxima: Critical Proximity (2016) de Tamara Díaz, el curador en jefe de TEOR/ética, ${ }^{1}$ Miguel A. López, concluye que el objetivo del libro es: "[...] reponer críticamente las condiciones de producción, así como las políticas del deseo, bajo la certeza de que son precisamente la proximidad y los lazos afectivos los que dotan de significado vital la manera en la cual construimos las historias e interpretamos la realidad" (López, 2016: 20).

Yfueronjustamente esas "políticas deldeseo" las que movieron al artista e informático costarricense Pablo Murillo, y a esta autora, a desarrollar Uncanny Valley Project para la X Sonora de la Bienal Centroamericana; en la videopresentación del proyecto se expone en los siguientes términos:

Explorar una heterotopía a través del sonido. Imaginar cómo es posible que la unión entre cuerpo y máquina, abra un espacio no-hegemónico en un "país"como San José. ${ }^{2}$ Pensar cómo la música electrónica puede traer "el futuro", ahora [...] cómo ciertos cuerpos pueden construir el futuro [...] una especie de distopía, quizás cibernética o de ciencia ficción, en una "ciudad"como San José, ahora.

¿Es eso posible? ¿Quiénes lo hacen posible a través de la música que crean, que construyen? ¿Cómo construyen su cuerpo, y el vínculo con la máquina y el sonido, creando esa heterotopía?

El "uncanny valley" como punto de partida o pretexto, para explorar estos cuerpos y su articulación, que en contexto resulta realmente inquietante. Nos ha de servir para documentar la configuración de estas personas, y al fin de cuentas generar una suerte de Quimera...un monstruo [... $]^{3}$

El proyecto se realizó en colaboración con Monik Zdan (DJ Monik), Coraima Díaz/Coco Nut Chan (Rompiste mis Flores), Susana López/Suzi Love (Bengalas), Fiamma Aleotti y Ronald Bustamante (Saturno Devorando), Señorita Abril, Natalia Ávalos Mora, Alison Alvarado, Sonya Carmona y Mari Navarro
(CoLoRnOiSe), Camila Garro y Paulina Velázquez, de Costa Rica. El proceso guiado por una "proximidad crítica" vinculó al proyecto a creadoras de otros países centroamericanos. Las redes sociales y los nuevos medios desterritorializaron la intervención, conectando a creadoras sonoras cuyas heterotopías generaban glitch (error) en los relatos sonoros acerca de Centroamérica y de la innovación y lo experimental en la región. Estas creadoras eran Tamara Montenegro y Clara Grun de Nicaragua, Mabe Fratti de Guatemala, Mar AlzamoraRivera de Panamá y Paola Moncada Pineda (Pasumopi) de Honduras, y Natalia Varela y Abigail Reyes de El Salvador (ver fotos 1 y 2 ).

En primer término, las personas implicadas en el proyecto compartían un tipo de resistencia hacia la "normatividad" del nacionalismo costarricense, sus retóricas "criollas" etnificantes y sus símbolos. Consecuentemente, ni el cancionero popular tradicional, ni los archivos etnográficos "indígenas" o “afrodescendientes”, ni los imaginarios sobre el país más feliz del mundo, el país verde o el paraíso ecológico y de paz, formaban parte de su trabajo. La disidencia estaba allí, en cómo compartían el rechazo a todo aquello, asumiendo por el contrario la distopía y filiación ciberpunk tanto en el trabajo de experimentación sonora, como en sus performances.

A este respecto, en el artículo "Ciberfeminismo y estudios sonoros", bajo el epígrafe "iSoñad ciborgs 'femeninos', 'queer' y sexys! iQue vuestro sonido electrónico reimagine la capital del país más feliz del mundo!", esta autora muestra cómo los cuerpos también narran y construyen imaginarios distópicos (Campos, 2016a: 151-158), en este caso a partir de la performatividad con la máquina —computer music, electrónica y noise — ${ }^{4}$ en comunión con el vestuario, el maquillaje, las puestas en escena, la producción audiovisual y el diseño de las portadas de álbumes - CD y EP-, ${ }^{5}$ remitiendo a distopías ciberpunk -inclusive sci-fi- ${ }^{6}$ y confrontando el canon de Costa Rica como oasis ecologista de paz.

Existe una comunidad dedicada a la música experimental y electrónica en el país, pero en este caso se trataba de mujeres, lo que marcó diferencias que se expondrán más adelante. En Costa Rica son los 
colectivos masculinos los que dominan el sector, y son ellos quienes controlan los espacios, las tecnologías y los discursos, tal y como se informa en el artículo ya mencionado (Campos, 2016a: 141-162).

Los colectivos masculinos experimentales costarricenses comparten estéticas derivadas del rock, el pop, el punk, la psicodelia y las culturas metal y hipster. Dicho así puede resultar simplista pero, en términos generales, en las producciones de aUTOperro, Extremos Sonoros y Oscilador, por ejemplo, intervienen individuos cuyo trabajo abreva de estas culturas, quienes expresan distintas maneras de hacer, vivir, construir y pensar (Campos, 2016a y en prensa). El artefacto y la performance están insertos en una manera de escuchar el mundo, y las genealogías de la escucha en estos casos remiten a las culturas citadas; queda pendiente un estudio de la construcción de masculinidades en estos colectivos.

Ahora bien, icómo pensar desde una proximidad crítica la comunidad en estudio? y icómo asumir la responsabilidad de construirla como "objeto de estudio"? Tanto Pablo Murillo como esta autora nos implicamos, él como fotógrafo ${ }^{7}$ y yo como compositora. ${ }^{8}$ Las creadoras sonoras con quienes se pretendía colaborar eran sujetos activos, no objetos; nosotras no constituíamos algo estable, pero estábamos construyendo algo. Algunas de las colaboradoras eran amigas, pero todavía no se habían propuesto crear algo juntas. El artista sonoro Ronald Bustamante era la figura bisagra entre nosotras, amigo común que nos conectaba, tal y como se explica en "Ciberfeminismo y estudios sonoros" (Campos, 2016: 148-151). La propuesta consistía en facilitar un espacio común de creación colectiva donde pudiéramos interactuar y producir juntas, donde compartir ideas y procesos. ${ }^{9}$

En síntesis, estábamos todas implicadas por la proximidad de afectos y por deseos comunes. Nos contaminábamos mutuamente, pero esa proximidad exigía a su vez un pensamiento que, como indica Tamara Díaz, ofreciera: “[...] una dimensión crítica, pero sobre todo poética del arte centroamericano [advirtiendo sobre] otros desplazamientos y énfasis en el arte contemporáneo de la región, comprendido en sus incidencias contextuales". A fin de cuentas, esta era una intervención para la X Bienal. Nosotras estábamos "tejiendo" algo que, como indica Díaz, incluía: "a la vez [...] el vínculo y la proximidad, la discontinuidad y la fragmentación, [...] aislamiento y transitoriedad" (Díaz, 2016: 24, 56), evidenciando una construcción, (de)construcción y (re)construcción de múltiples identidades (2016: 48-49). Un ejemplo es cómo las creadoras en estudio construían personajes y performances, los cuales se conservan en las fotografías de Murillo.

¿Qué proximidad íbamos a pensar, a mostrar? Sabíamos que, como indica Díaz, "[...] si revisamos las exposiciones y publicaciones en torno a Latinoamérica que empezarían a proliferar desde los noventa en los circuitos centrales, es evidente la ausencia casi absoluta de artistas centroamericanos". La curadora se pregunta: "iLa construcción de Centroamérica en el campo artístico fue tal vez una contestación a esos vacíos? ¿Un gesto de vaga correspondencia a la demanda de 'regionalismos' de los centros?" (Díaz, 2016: 122). El propósito de Uncanny Valley Project era dialogar con estos cuestionamientos, especialmente, volviendo a Díaz, “[...] preguntar en cuál Centroamérica es pensada o imaginada y cuándo constituye sólo un telón de fondo o un conveniente marco institucional" (2016: 121).

El proyecto de intervención en la X Sonora de la Bienal concebía críticamente los regionalismos al cuestionar su normatividad, y pensaba en la comunidad en que se insertaba. La X Sonora era una especie de "comunidad imaginada musicalmente" (Born, 1993: 286) que reunía personas invitadas por la Bienal para intervenir en representación de su país de origen. Esta representatividad era cuestionada por la no-normatividad del proyecto, que se autodenominaba ciberfeminista. Pero, ¿cómo se vinculaba el ciberfeminismo con la proximidad crítica? Se vinculaba a partir de la propuesta curatorial Todas las vidas de Tamara Díaz, y cito:

Todas las vidas cuestiona los límites de vidas humanas y no humanas, humano y animal, naturaleza y cultura. Junto al feminismo, comprende la vida en términos 
de interdependencia, en su condición precaria y vulnerable. ¿Qué podemos aprender de poéticas basadas en procesos orgánicos, de obras que duran, que huelen, que se transforman, que mueren? ¿Qué potencia de contagio movilizan redes, plagas y enjambres? ¿Y cómo contagiar que todas las vidas importan? (Díaz, 2016b).

La propuesta de Díaz coincide con el Manifiesto Cyborg de Donna Haraway en su búsqueda de quimeras, y cito un fragmento del Manifiesto: "[...] todos somos quimeras, híbridos teorizados y fabricados de máquina y organismo, en una palabra, somos ciborg" (1995: 254). Consecuentemente, ciberfeminismo y proximidad crítica se aunaban en esta intervención al experimentar con la hipótesis del uncanny valley (valle inquietante), propuesta por Masahiro Mori en los años setenta. Uncanny Valley Project sintonizaba con la "reinvención de la naturaleza" que promulgaba la propuesta curatorial de Tamara Díaz. Nosotras la subrayamos con el nombre del proyecto, remitiendo a: "[...] the hypothesized that a person's response to a humanlike robot would abruptly shift from empathy to revulsion as it approached, but failed to attain, a lifelike appearance. This descent into eeriness is known as the uncanny valley" (Masahiro, MacDorman y Kageki, 2012).

La hipótesis del "valle inquietante" se convertía en una pregunta de investigación artística para nosotras, al identificar en estos términos la anomalía que representaban las creadoras sonoras en la escena de la música electrónica y los relatos acerca del arte centroamericano. Ellas eran criaturas "inquietantes" frente a los panteones patriarcal-nacionalistas que dominan los cuerpos, el discurso, las tecnologías y los espacios de la creación sonora y musical hasta la actualidad. Era la primera vez que estas creadoras realizaban un proyecto juntas, construyendo "una habitación propia" — cabe aquí citar a Virginia Woolf-, donde el noise, la música electrónica, el arte sonoro y la música experimental que producían tendrían visibilidad en el marco de la Bienal.

Uncanny Valley Project procuraba explorar, desde el ciberfeminismo, las relaciones entre cuerpo y máquina, performance y procesos creativos, considerando, como indica Octavio Spíndola Zago, la posibilidad de:

[...] los no lugares [como] catalizadores epistémicos de heterotopías, lugares que - siguiendo a Foucault (2010: 19-32) - yuxtaponen espacios; son recortes geográficos de tiempo, sistemas de aislamiento a partir de la gestión territorial corporalizada, reinvenciones rizomáticas objetivadas que se cierran y abren al exterior, generan experiencias traumáticas a la vez que renovadoras, son un horizonte de posibilidades, de renarrativización espacial, y consienten directrices políticas revolucionarias o, al menos, transformadoras (Spíndola, 2016: 30).

En estos términos, las creadoras en estudio resultaban inquietantes. Su existencia, su creación, su performatividad, constituían una heterotopía. Uncanny Valley Project como proceso pretendía explorar esta posibilidad a partir del ciberfeminismo y la proximidad crítica, primero a nivel costarricense y, conforme la intervención entró en acción, a nivel centroamericano.

\section{La proximidad crítica de una comunidad imaginada}

Una intervención ciberfeminista en la X Sonora de la Bienal Centroamericana, ipor qué? Explicar este punto requiere retomar "Ciberfeminismo y estudio sonoros" (Campos, 2016: 141-162). Tal y como informé en ese artículo, existe un activismo vinculado con la creación experimental de artistas sonoras y compositoras. Actualmente estas comunidades ciberfeministas se relacionan creando grupos en redes sociales como "Women in Experimental", "Mujeres en la experimentación sonora // Latinoamérica", "11 "FFF"12 o páginas como "Musicología feminista"13 y "Museroule - Women in experimental music". ${ }^{14}$ También organizan su trabajo bajo la curaduría de diferentes agentes en archivos especializados como "Her Noise Archive", 15 "Feminatronic" ${ }^{16}$ o "female: pressure", 17 y en proyectos y programas dedicados a fomentar su trabajo como "Women in Electronic Music" del Canadian Music 
Centre, ${ }^{18}$ "Femke Cult. Modern Perspectives in Art, Music \& Culture"19 o LA BIONICA, "espacio de difusión inserto en el Festival INTERFACE Arte, Cuerpo, Ciencia y Tecnología que tiene como objetivo difundir el trabajo de mujeres artistas e investigadoras latinoamericanas que desarrollan y manufacturan sus propios objetos, herramientas y propuestas en arte-tecnología". ${ }^{20}$ También destacan publicaciones monográficas como Pink Noises: Women on Electronic Music and Sound, de Tara Rodgers, y Women of the Underground: Music:Cultural Innovators Speakfor Themselves, editado por Zora von Burden, ambos de 2010.

Estos son algunos ejemplos de tipos de activismo que a su vez están vinculados a proyectos nacionales, regionales e internacionales, como "Microcircuitos. Plataforma independiente de experimentación sonora generada desde América Latina", ${ }^{21}$ donde Costa Rica tiene su propio espacio dentro del cual, al día de hoy, sólo se incluye a una compositora (Susan Campos), ${ }^{22}$ y al espacio DEBATES SONOROS-UCR que la autora de este artículo coordina en la Universidad de Costa Rica (UCR). ${ }^{23}$

A este respecto, el compositor y artista sonoro costarricense Otto Castro, editor de Microcircuitos Costa Rica, fundador del Laboratorio de Composición y Experimentación Sonora (CES) de la UCR ${ }^{24}$ y miembro fundador de la Red de Arte Sonoro Costarricense Oscilador, señaló al periodista Vinicio Chacón en el artículo "iArtistas sonoros uníos!" que: "El proyecto también se interesa por encontrar compositoras activas en la región". Para Castro, "en Costa Rica son invisibilizadas", aunque sí destacó el aporte que han hecho desde fuera del ámbito de la composición académica artistas como Mariela Richmond, Paulina Velásquez ${ }^{25}$ o Susan Campos" (Chacón, 2013). El interés de la plataforma Microcircuitos por localizar compositoras y artistas sonoras es un indicador más de los activismos mencionados.

Este tipo de activismo encuentra en el área de las humanidades diferentes espacios académicos para su inclusión, uno de los cuales es la llamada "musicología feminista”. Ahora bien, los vínculos entre feminismo y musicología se han materializado en diversos trabajos, proyectos y publicaciones, fundamentalmente relacionados con los estudios sobre las mujeres. ${ }^{26}$ No obstante, cuando se planteó en la Society of Music Theory, tal y como quedó reflejado en el dossier "Toward a Feminist Music Theory" publicado en Perspectives of New Music (VV.AA., 1994), lo que se propuso fue un proyecto de teoría musical que abogara, muy grosso modo, por la diversidad en la investigación feminista, el estudio de performances sociales, diferentes tipos de embodiment e empowerment, el análisis del cuerpo como parte de la experiencia musical y las contradicciones de la experiencia generizada, en resumen, como "political projects of feminist criticism" (1994: 6-7); no sólo se abocaba a la identificación de creadoras.

En sintonía con este objetivo, en 2011 coedité junto al Dr. Josemi Lorenzo un dossier especial en la revista Transcultural de la Sociedad Ibérica de Etnomusicología, en celebración de Feminine Endings. Music, Gender, and Sexuality (1991) de la Dra. Susan McClary, uno de los libros fundadores de la llamada "musicología feminista" (2011). Sin embargo, dada la predominante filiación de los estudios de género con los estudios sobre las mujeres en los estudios musicológicos en lengua castellana, ${ }^{27} \mathrm{el}$ resultado fue un conjunto de estudios que titulamos Música y estudios sobre las mujeres (Campos y Lorenzo, 2011). Y esto, a pesar de que la Dra. McClary señaló en su artículo para el dossier la necesidad de una reflexión crítica sobre esta filiación ante el propio objetivo de Feminine Endings, que procuraba explorar las relaciones entre teoría feminista y teoría musical en un sentido amplio (McClary, 2011).

Retomando este objetivo, la Dra. Isabel Porto Nogueira y esta autora coeditamos en 2013 el libro Estudos de gênero, corpo e música: abordagens metodológicas, publicado por la Associação Nacional de Pesquisa e Pós-Graduação em Música (ANPPOM) en la Série Pesquisa em Música no Brasil (Campos y Porto, 2013). Este trabajo sí consiguió ampliar el espectro de perspectivas teóricas de análisis, pero los estudios sobre las mujeres siguieron siendo dominantes. Esto nos motivó a proponer un segundo volumen - aún en fase conceptual-, donde abordar críticamente el porqué de este dominio, explorándolo desde activismos 
feministas de diversa índole dentro de la creación experimental. El proyecto aún es sólo una idea, de la cual forma parte este testimonio como una iniciativa en sí misma experimental, de posibles vinculaciones -al parecer aún demandadas y demandantes-, para pensar una comunidad que evidentemente existe, y se imagina, desde la proximidad crítica entre teoría feminista, teoría musical y creación. Uncanny Valley Project es, consecuentemente, al igual que "Ciberfeminismo y estudios sonoros", un salir en busca de "new feminine beginnings", retomando la conclusión a la que llegó la Dra. McClary en su declaración de 2011.

\section{Autoetnografía e investigación artística}

En los siguientes apartados se describirá y analizará en qué consistió el proceso de la intervención ciberfeminista Uncanny Valley Project en la X Sonora de la Bienal Centroamericana 2016.

\section{El proceso, fase 1}

La X Bienal otorgaba al proyecto un plazo de veinte días para intervenir en sus redes - del 10 al 30 de mayo de 2016 - intervención que consistía en subir contenidos de audio y video a sus redes (Youtube, Spotify y SoundCloud), y socializarlos junto a otros materiales afines a través de usuarios personales en redes sociales (Facebook, Twitter, Google plus e Instagram). Se utilizó con este propósito el hashtag \#UncannyValleyProject, que se incluía en cada post publicado.

Los materiales a compartir se dividieron en:

1. artículos vinculados con creadoras sonoras a nivel internacional,

2. audios de producciones individuales de las colaboradoras en el proyecto,

3. audios de producciones colaborativas realizadas en el marco del proyecto,

4. playlist: una para Costa Rica y otra para Centroamérica,

5. fotografías realizadas por Pablo Murillo,
6. fotografías que documentaban las sesiones de trabajo del proyecto,

7. fotografías de eventos vinculados o vinculantes,

8. información sobre actividades científicas donde se compartía el proceso de la intervención, a saber, el Encuentro Feminismos, Géneros y Comunicación, ${ }^{28}$ y el I Encuentro sobre Estudios Sonoros y Creación Experimental, ${ }^{29}$ ambos celebrados durante el mes de mayo, organizados por la UCR y

9. textos cortos en el blog de esta autora. ${ }^{30}$

Los rastros y la gestión de la intervención pueden seguirse a través del hashtag. En relación con el primer tipo de documentos, los artículos sobre creadoras sonoras a nivel internacional introducían en un contexto, evidenciaban cómo la intervención coincidía con una preocupación y un área de estudio, algo fundamental para ese "tejido" que se procuraba materializar. Este procedimiento fue especialmente útil frente a los receptores locales, que dejaban comentarios como: "la música no tiene género", "una obra vale por sí misma, la música que es buena, es buena". Un ejemplo es el comentario de José Duarte, artista sonoro costarricense - uno de los líderes de Extremos Sonoros-, quien escribió en el muro de su perfil de Facebook, acompañando el texto con un enlace a TRON RUN/r Game Rip Soundtrack, la siguiente recomendación:

La vara no es Cyber Feminismo ni inventarse otro término. Ahora es el come back del Cyber-Punk y es lo que vivimos ahora... es la realidad: el sistema nos quiere consumir, es hora de comprar oro y hacer canjes de bitcoins. Quedar fuera del sistema y explorar a los vedas. /// dejarse de varas es lo que sigue $[. . .]^{31}$

El comentario anterior se publicaba luego de que la intervención compartiera en Facebook el videoclip Time de CoLoRnOiSe, ${ }^{32}$ en el que se incluía esta pregunta:

¿Un imaginario cyberpunk, distópico y apocalíptico "centroamericano"? CoLoRnOiSe Alison Alvarado 
+ Sonya Carmona nos dan una muestra en "Time" (2013), un ejemplo magnífico de la heterotopía que \#UncannyValleyProject explora para la Bienal Centroamericana. Hemos querido rendir homenaje a estas dos grandes creadoras sonoras costarricenses, incluyéndolo en nuestra intervención para X SONORA..$^{33}$

¿Una coincidencia? Definitivamente no. La crítica iba dirigida a la conceptualización del proyecto, que había sido presentado como ciberfeminista en los medios de la Bienal, y a la elección de un videoclip de CoLoRnOiSe como representante de una perspectiva ciberpunk, anómala y disruptiva en Centroamérica. El autor del comentario, con su recomendación alternativa, hacía referencia a narrativas heroicas masculinas y patriarcales de ficción que encontraba en los personajes de videojuegos como TRON RUN/r. No está de más señalar que tanto el videoclip de CoLoRnOiSe, como el videojuego TRON RUN/r, se basan en sagas de persecución. Tampoco sobra recordar la tradición misógina de los videojuegos de acción y competición. Para nosotras, el valle inquietante quedaba manifiesto aquí, en la recepción de una figura como José Duarte, destacado e influyente artista sonoro costarricense, quien posiblemente manifestaba un malestar gremial ante esta irrupción, quizás ante el hecho de que fuera un proyecto de creadoras - mujeres - el que representara a Costa Rica en la X Sonora de la Bienal. Algo histórico, ya que hasta el momento este ha sido un dominio de compositores y artistas sonoros.

En resumen, los artículos que compartimos en redes antes, durante y después de la intervención cumplían su objetivo al demostrar una preocupación internacional por la brecha de género en la creación experimental y la música electrónica. Algunos ejemplos son: la "editatona" convocada por MediaLab Prado (España); ${ }^{34}$ playlists dedicadas a las pioneras de la música electrónica, como la publicada por la revista española El Estado Mental; 35 un video publicado por la revista PlayGround (España) donde se expone la brecha en el mercado y los festivales de "música avanzada"; 36 artículos como "Mothers of Invention: the Women Who Pioneered
Electronic Music” de Jennifer Lucy Allan en el periódico The Guardian (Allan, 2016), "Sound::Gender::Feminism:: Activism: Research and Challenges to the Orthodoxies of Sound Arts" de Cathy Lane en Contemporary Music Review (Lane, 2016: 32-39), "Women and their Machines: A Think-piece about Female Pioneerism in Electronic Music, Post-post Feminism and Some Sassy Statements on Sexism” de Katrin Richter en Meoko.net (2013), "DJ Maria Chavez Embraces Beautiful Destruction on the Turntable" de Sophie Weiner en The Village Voice (2016) y "18 Women in Electronic Music You Need to Hear Right Now" de Ryan Creed en BuzzFeed (2016); proyectos como Women in Sound/Women on Sound del Lancaster Institute for the Contemporary Arts de Lancaster University, ${ }^{37}$ y festivales como SHE MAKES NOISE coordinado por Natalia Piñuel (España). ${ }^{38} \mathrm{Al}$ socializar en redes estos materiales, procuramos comunicar al público local que ésta no era una acción aislada, caprichosa y sin criterio, ${ }^{39}$ sino un proyecto riguroso que tejía redes desde Costa Rica hacia Centroamérica, sintonizando con improntas afines a nivel mundial.

Coincidiendo con Georgina Born y David Hesmondhalgh, estábamos pensando, "[...] musicallyimagined communities, and music that is driven by sociocultural identities that are ontologically and sociologically prior, even if their enhancement and enactment in musical practice and performance produces effects on those identity formations" (Born y Hesmondhalgh, 2000: 16-17). En el marco de la X Bienal se evidenciaba, así, una comunidad musicalmente imaginada que existía en proximidad crítica, con todo lo que esto tiene de "pacto de sangre", de (auto)exploración y (auto)experimentación. Consecuentemente, para sistematizar la intervención se eligió como área de trabajo la investigación artística y, como método, la autoetnografía.

Uncanny Valley Project era una intervención premeditada, era parte de un proyecto de investigación, ${ }^{40}$ yera un experimento. Tenía como propósito involucrarse activamente en el problema de estudio, ser parte del mismo. Esta autora había estudiado la comunidad de compositores y compositoras de "música de arte" —académica - y a las mujeres y hombres creadores 
sonoros experimentales — no académicos- de Costa Rica durante varios años. ${ }^{41}$ Los resultados de esta investigación evidenciaban la necesidad de realizar una acción más radical, era necesario abrir una brecha, acercarse e implicarse con la comunidad en estudio, y a esta acción Tamara Díaz le da nombre cuando habla de "proximidad crítica".

Se procedió entonces a organizar el experimento dentro de un área, y para ello se eligió la investigación artística. Esto también tenía un contenido crítico ya que en el país, desde la inauguración del Instituto de Investigación en Arte de la UCR en 2013, el debate acerca de qué es exactamente "eso" y cómo funciona parece ser una prioridad para la institución universitaria y los profesionales en prácticas artísticas, sus tesis de posgrado y proyectos de investigación. A continuación se consideró una metodología; se eligió la autoetnografía porque ésta facilitaba un tejido permeable, poroso, entre la acción sistémica y el acto testimonial. Se diseñaría, consecuentemente, un plan básico que diera seguimiento a las acciones y considerara imprevistos, incorporándolos al proceso de manera flexible.

Rubén López Cano y Úrsula San Cristóbal, en su guía didáctica Investigación artística en música. Problemas, experiencias y modelos (2014), ofrecen una esquemática descripción de funciones, tipos, estrategias, métodos y técnicas autoetnográficas (López Cano y San Cristóbal, 2014: 135-183). Para esta intervención resultó fundamental el apartado dedicado a la proximidad, al enfocarla desde el querer y el deseo, coincidiendo con lo expuesto por Tamara Díaz y Miguel A. López (López Cano y San Cristóbal, 2014: 178). El diseño de la intervención en la X Sonora siguió las recomendaciones de esta guía basándose en cuatro preguntas básicas: 1) ¿qué queremos experimentar?, 2) ipara qué queremos hacerlo?, 3) icómo queremos hacerlo? y 4) icómo evaluar los resultados?

Estas preguntas facilitaron el diseñoy la planificación experimental de Uncanny Valley Project. Para organizar el proceso, las acciones y variables, metas y fines, serían objetuables (con objetivos), procesuales (con procedimientos) y conceptuales (con ideas). La intervención tenía una fecha de inicio y de conclusión, y el diseño y la planificación debían responder a estas condiciones para conseguir los objetivos propuestos, considerando imprevistos.

\section{Elproceso, fase 2}

La primera pregunta a responder era: ¿qué quería experimentarse? La intervención procuró experimentar con la hipótesis del uncanny valley (valle inquietante) propuesta por Masahiro Mori en los años setenta (Kageki, 2012). Esta hipótesis sería aplicada al estudio de las creadoras sonoras costarricenses que utilizaban medios electrónicos — analógicos y digitales - en su proceso creativo. La elección de esta hipótesis estaba vinculada con la performatividad y las propuestas - la obra sonora- de las creadoras identificadas, quienes mostraban una tendencia a estéticas futuristas, sci-fi y cyberpunk vinculadas con la robótica. La intervención tomó en consideración posteriormente a creadoras sonoras centroamericanas. En este caso las tendencias no eran tan homogéneas como en el caso costarricense; sin embargo, se mantuvo la hipótesis considerando como base la performatividad del cuerpo con la máquina (computer music) y la estética techno vinculada con la música electrónica de baile. La exploración de la hipótesis tenía como objetivo estudiar la heterotopía, el no-lugar que estas creadoras diseñan y piensan para sí mismas en la escena de la música electrónica.

En cuanto a la segunda pregunta, ipara qué quería hacerse?, la intervención en la X Sonora de la Bienal proponía algo muy puntual. Fundamentalmente solicitaba compartir material audiovisual en las redes y medios dispuestos para ello por la Bienal. En resumen, solicitaba un producto que podía ser una obra musical, una pieza de arte sonoro, de paisaje sonoro o de poesía sonora, un videoclip, una pieza de videoarte, imágenes y enlaces vinculados. Los canales disponibles, como se indicó anteriormente, eran Youtube, Spotify, SoundCloud, Facebook, Twiter, Google plus e Instagram. Uncanny Valley Project estaba pensado como un proyecto colectivo; no obstante, por disposición de la curadora jefe de la Bienal, sólo una persona podía tener acceso a las claves de las redes de la X Sonora, 
quien asumiría la responsabilidad de desarrollar la intervención, elegir los contenidos, construir el discurso y compartir los productos resultantes. Esta autora asumió dicha responsabilidad.

Continuando con la respuesta a la pregunta relacionada con para qué quería hacerse, la X Sonora tenía un propósito básico: posibilitar espacios de socialización, creación y visibilización para las creadoras sonoras. Ahora bien, ¿cómo se vinculaba este propósito con la hipótesis y los objetivos de la investigación?, a saber, iqué se quería experimentar? Era evidente que se planteaba un problema experimental que superaba lo que solicitaba la intervención en la X Sonora de la Bienal. La hipótesis del uncanny valley se quedaría en metáfora, y esto debía ser considerado al analizar los resultados.

Tomando esto en cuenta, la intervención podría servir como una primera aproximación experimental al problema propuesto, lo que determinaba el tercer paso: ¿cómo quería hacerse? Teníamos la oportunidad de realizar una intervención capaz de evidenciar que dentro del arte centroamericano existía el trabajo de creadoras dedicadas al arte sonoro, el noise y la música electrónica y electroacústica, que no conformaban una comunidad, pero que se reunían con el objetivo de posibilitar una, aunque fuese de manera transitoria. La intervención en sí misma era una heterotopía.

La X Sonora de la Bienal Centroamericana era un espacio innovador — era la primera vez que se incluía una sección como ésta en la Bienal—, su propósito era dar lugar a la investigación, creación y experimentación sonora producida por artistas de la región, incluyendo algo más que las "instalaciones sonoras" de las artes visuales. Uncanny Valley Project era además la intervención que representaría a Costa Rica. Todo esto debía ser tomado en cuenta al diseñar el plan de trabajo para que fuera acorde con las condiciones y limitaciones identificadas.

Dado que la X Sonora solicitaba un producto, se incluirían obras individuales de cada una de las colaboradoras y se realizarían dos sesiones de trabajo para crear las obras colectivas. Esto último cumpliría con el objetivo de facilitar un espacio de socialización entre pares. La elección de los espacios para las sesiones debía ser en sí misma una intervención política acorde con los objetivos del proyecto. Consecuentemente, se eligieron dos espacios, el de "la academia", representado por la Escuela de Artes Musicales de la UCR —donde esta autora es docente- y el de los artistas sonoros - hombres - representado por el estudio de nuestro colaborador Ronald Bustamante.

Las obras individuales y colectivas fueron incluidas y organizadas como playlist en el SoundCloud de la Bienal. Paralelamente, con el propósito de mostrar cuáles eran las referencias personales de las colaboradoras, se generaron playlists con sus top ten en Spotify. También se generaron sinergias con redes personales: Coraima Díaz invitó a la bailarina Jackie Zampalioni y a la diseñadora Ariadna Poupee Bla, y esta autora a la clavecinista María Clara Vargas, actual decana de la Facultad de Bellas Artes de la UCR. Las obras individuales sumaron en algunos casos colaboraciones de artistas sonoros y músicos, específicamente de Alejandro Sánchez Núñez (Begotten Costa Rica), Ronald Bustamante, Revuelta Sonora y la banda Amelia. Las colaboraciones con artistas sonoros no fueron descartadas, considerando la autoría individual o colectiva de las creadoras. No obstante, como indicaron posteriormente Fiamma Aleotti (Saturno Devorando) y Madame Bowman (Señorita Abril) en Sonora Desconferencia (2 de noviembre de 2016), esto puede dar lugar al debate, ya que al formar parte de un grupo o banda suele asumirse que la autoría es del creador varón, no de la mujer. ${ }^{42}$

Finalmente, las fotografías de Pablo Murillo que se eligieron como imagen del proyecto muestran el cuerpo desnudo de una mujer. Esta elección procura evidenciar cómo la desnudez de las mujeres se vincula con la explotación sexual y la pornografía, no con la innovación y la experimentación en la creación sonora. La tradición canónica en el "mundo de la música" fomenta la visión del cuerpo desnudo de la mujer como acompañamiento al genio varón, sea como musa, amante, esposa, madre, etcétera. El propósito de las fotografías de Murillo era conectar con esta tradición según la cual el cuerpo de las mujeres se objetualiza y, al mismo tiempo, reivindicar con esta acción un empoderamiento corporal. La portada de 
Uncanny Valley Project Costa Ricafue "The Usual Amount of Damage" (2015), yla de Uncanny Valley ProjectCentroamérica incluía un detalle de "La Inmaculada Conceptual" (2015). En ambas fotografías se mostraba el cuerpo de una mujer con su vulva sin depilary, en el segundo caso, se incluía el detalle de una pequeña estatua de la Virgen María. Esta acción crítica iba dirigida al culto mariano predominante en la región centroamericana y a sus repercusiones en la construcción de estereotipos sobre las mujeres (ver foto 3$)$.

A continuación se desglosa el plan de trabajo:

a. Sesión de trabajo I en la Escuela de Artes Musicales (9 de abril de 2016), utilizando nuevos medios electrónicos y un piano de media cola. Participantes: Coraima Díaz, Monik Zdan, Fiamma Aleotti y Susan Campos. Resultado: dos obras colaborativas: "R\&B 12 MD" (Susan Campos y Monik Zdan) y "Taste It" (Susan Campos y Fiamma Aleotti + Ronald Bustamante/ Saturno Devorando).

b. Sesión de trabajo II, en el Studio de Ronald Bustamante (29 de abril de 2016). Participantes: Coraima Díaz y Susana López. Resultado: la obra colaborativa "Volverte a ver".

c. Colaboración con la clavecinista María Clara Vargas y con el artista sonoro Ronald Bustamante para grabar y estrenar la obra "Esfinge" de Susan Campos. La obra se grabó el 3 de mayo de 2016 y se estrenó en concierto ClaveciNOISE el 25 de mayo de 2016 en el marco del I Encuentro sobre Estudios Sonoros y Creación Experimental celebrado en la UCR. ${ }^{43}$

d. Desarrollar la playlist Uncanny Valley Project CostaRica en el SoundCloud de la Bienal, publicada el 10 de mayo de 2016, ${ }^{44}$ que incluye las siguientes obras:

1. "Volverte a ver", de Coraima Díaz y Susana López.

2. "R\&B 12MD”, de Susan Campos y Monik Zdan.

3. "Taste It", de Susan Campos y Fiamma Aleotti + Ronald Bustamante (Saturno Devorando).
4. "Hollows Humans", de Paulina Velázquez.

5. "Esfinge", de Susan Campos, María Clara Vargas y Ronald Bustamante.

6. "Sad Song", de Fiamma Aleotti y Ronald Bustamante (Saturno Devorando).

7. "She Was Machine", de Alison Alvarado, Sonya Carmona y Mari Navarro (CoLoRnOiSe).

8. "Butoh Meditation \#3-Prophecy", de Susan Campos y Begotten Costa Rica.

9. "Dangerous Women", de Susan Campos.

10. "Cómo morir [ahogada]", de Fiamma Aleotti.

11. "Butoh Meditation \$2-Espino blanco", de Susan Campos y Begotten Costa Rica.

12. "Aleph", de Señorita Abril y Saturno Devorando.

13. "Ex Mistake", de CoLoRnOiSe.

14. "Transmission Synaptique", de Fiamma Aleotti.

15. "Periplo azul", de Camila Garro y Ronald Bustamante.

16. "Dark Thoughs", de Coraima Díaz.

17. "Demons Tears", de Coraima Díaz.

18. "Live at El Sótano", de Susan Campos.

19. "Human Feeling", de Saturno Devorando.

20. “Apple - Intention”, de Natalia Ávalos.

e. Desarrollarlaplaylist Uncanny Valley ProjectCentroamérica en el SoundCloud de la Bienal, publicada el 15 de mayo de $2016,{ }^{45}$ que incluye las siguiente obras:

1. "Propósito A-literal", de Tamara Montenegro.

2. "Piano for Tamara", de Clara Grun.

3. "Síntoma", de Mabe Fratti.

4. "Sala Bipolar", de Clara Grun y Revuelta Sonora.

5. "Lethe", de Mabe Fratti.

6. "Live @ Semana de la Música Avanzada Guatemala 2016", de Tamara Montenegro.

7. "Ol Jardìn", de Mar Alzamora-Rivera y Amelia.

8. "Sin Retorno", de Mar Alzamora-Rivera.

9. "NVarela-Felipe", de Natalia V (Sister Natty).

10. "Alegría tiene nombre de mujer", de Abigail Reyes. 
11. "Amicizia (Auricula remix)", de Tamara Montenegro.

f. Solicitar playlists con los top ten de cada participante para publicarlas en Spotify. ${ }^{46}$

g. Realizar una intervención en el canal de Youtube de la Bienal. ${ }^{47}$ Se incluyen los siguientes videoclips:

1. Presentación del proyecto, a cargo de Susan Campos y Pablo Murillo.

2. "R\&B12 MD", con música de Susan Campos y Monik Zdan, y dirección artística de Coraima Díaz en colaboración con Natalia Ávalos, Jackie Zampalioni y Ariadna Poupee Bla.

3. "Aleph" (Improvisesiones con ElectroJuguetitos), de Señorita Abril y Saturno Devorando.

4. "Glenn Gould", de Saturno Devorando.

5. "Little Black Angel", de Saturno Devorando.

6. "Time", de CoLoRnOiSe.

7. "Esfinge", de Susan Campos.

8. "Las mieles de los cyborgs", de Pablo Murillo.

9. "Noise: filosofía y música en disputa", conversatorio realizado en la Escuela de Filosofía de la UCR, organizado por Pablo Hernández, que contó con la participación de los expositores Susan Campos Fonseca, Sergio Wiesengrund, Frank Granados y de los músicos Ronald Bustamante Medina, José Manuel Rojas González y Alejandro Sánchez (Achromatic Prods-Alejo SN).

El desarrollo de las playlists de Uncanny Valley Project Costa Rica y Uncanny Valley Project Centroamérica fue el resultado de un ejercicio curatorial realizado por esta autora bajo las premisas del proyecto. Se consideraron obras disponibles en SoundCloud y Bandcamp. También se solicitaron obras originales que aparecen en las playlist bajo el usuario de la Bienal. La selección de los videos compartidos en el canal de Youtube sólo incluye casos costarricenses: Señorita Abril, Saturno Devorando y CoLoRnOiSe. También se organizó un álbum con fotografías de
Pablo Murillo, que incluía sus retratos de las creadoras participantes, fundamentalmente de Señorita Abril, Saturno Devorando y Bengalas. El álbum funcionó a su vez de bitácora al incluirse fotografías realizadas por las participantes durante las sesiones de trabajo y afiches de eventos vinculados con la participación en actividades científicas, conciertos y entrevistas. También se dio seguimiento a actividades posteriores a la intervención que podrían considerarse consecuencias de la propuesta. Un ejemplo es el evento Juno, realizado por Monik Zdan, Coraima Díaz y Ronald Bustamante, el 16 de junio de 2016 en Amón Solar (San José, Costa Rica), ${ }^{48}$ y Sonora Desconferencia, organizada en el II Encuentro sobre Estudios Sonoros y Creación Experimental (ver foto 4).

En términos generales, el plan de trabajo permitió organizar la intervención, realizando cierto proceso curatorial bajo la hipótesis propuesta. En retrospectiva, un análisis del proceso a nivel de producción coincide con la hipótesis de Masahiro Mori en la medida en que, y cito: "[...] the concept of the uncanny valley has rapidly attracted interest in robotics and other scientific circles as well as in popular culture" (Mori, MacDorman y Kageki, 2012). No obstante, a nivel teórico la intervención ciberfeminista utilizaba la hipótesis de manera invertida al explorar el fenómeno de la performatividad y la estética robótica practicadas intencionalmente por humanos, en este caso, dedicados a la música electrónica en sus diferentes manifestaciones. En el caso de la población en estudio, se examinaba cómo esta performatividad se vincula con el ciberpunk y la ciencia ficción, incluyendo también la representación de "lo alienígena".

Ejemplos destacados a nivel canónico de este fenómeno son la banda alemana Kraftwerk, fundada en 1970, el músico y compositor británico David Bowie (1947-2016), la cantante, modelo y actriz jamaiquina Grace Jones (1948), la cantautora, compositora y productora islandesa Björk (1965), o las estadounidenses Holly Herndon (1980), artista sonora, y Janelle Monáe (1985), cantante y productora, entre otros. ${ }^{49}$ Consecuentemente, un resultado concluyente de Uncanny Valley Project fue identificar dicho fenómeno 
al interior de la música electrónica costarricense y, a partir de la intervención en la X Sonora de la Bienal, darle visibilidad dentro del arte centroamericano subrayando el aporte de las creadoras. La intervención ciberfeminista coincide así con la propuesta curatorial Todas las vidas de Tamara Díaz, al cuestionar "los límites de vidas humanas y no humanas, humano y animal, naturaleza y cultura" (Díaz, 2016), guiada en su proceso por la hipótesis del uncanny valley.

\section{Recepción de la intervención ciberfeminista}

Las sinergias creativas programadas produjeron los resultados previstos: tres colaboraciones originales para el proyecto, aunque lo óptimo hubiera sido conseguir al menos una obra colaborativa por autora, es decir, cinco obras, considerando que cinco constituían el 10\%, y tres da un aproximado de un 8\%, cifra aceptable. El programa radial en línea The Recognition Test dedicado a la curaduría de música experimental a nivel internacional incluyó "Taste It" (Susan Campos y Saturno Devorando), en su selección semanal, el 15 de mayo de $2016^{50} y$, posteriormente, el 7 de enero de 2017 el curador sonoro Raffaele Pezzella incluyó "Taste It" en el álbum Difference - visions of contemporary composition, considerándola entre las obras más representativas del año 2016 en este ámbito. ${ }^{51}$

En el programa Artiquicia de Radio U de la UCR, su conductora Amanda Rodríguez realizó una entrevista en vivo a las colaboradoras costarricenses el 18 de mayo de 2016. Se mencionó el proyecto en la prensa local, específicamente en los artículos "Susan Campos: inquieta creadora de sonidos", de Fernando Chávez-Espinach (2016), y "La X Sonora lleva la Bienal Centroamericana a sus audífonos", de Carlos Soto Campos (2016).

En su artículo, Chávez-Espinach escribe: "Actualmente, Campos [...] participa en la Bienal Centroamericana - quededicaX Sonora a la exploración en ese campo en la región-. Con el Uncanny Valley Project se reúnen creadoras como Fiamma Aleotti y Coraima Díaz; se publicarán un álbum colectivo y un libro" (Chávez-Espinach, 2016).
En su artículo, Carlos Soto escribe:

En mayo, la compositora costarricense Susan Campos reunió en el proyecto Uncanny Valley los trabajos de compositoras, Dj e investigadoras sonoras de la región.

"Decidí no hablar de mi obra, sino aprovechar la oportunidad para crear una red entre las creadoras centroamericanas, hacer lo que llamo una intervención ciberfeminista", expresó Campos por vía telefónica. [...] En la opinión de Campos, la X Sonora es una oportunidad para revisar el trato que tiene lo sonoro desde el mundo del arte. [... En Costa Rica] la gente que hace curaduría aún no está preparada para trabajar con lo sonoro; lo dejan aparte, sigue siendo un terreno oscuro", expresó Susan Campos.

Tamara Díaz (curadora de la Bienal), abrió una puerta importantísima para discutir, intercambiar bagajes teóricos y crear redes en la región", finalizó (Soto, 2016).

Respecto al libro y el álbum colectivo que indica Chávez-Espinach, todavía son un proyecto abierto, y este testimonio es parte de ese proceso, requerido por toda investigación. Lo que se describe en este texto es resultado de una primera reflexión académica sobre la experiencia, compartida en el marco de la Cátedra Mercedes Olivera del Programa de Doctorado y Maestría en Estudios e Intervención Feministas de la Universidad de Ciencias y Artes de Chiapas (Campos, 2016b).

No obstante, todavía existe un silencio en torno a la sección X Sonora de la Bienal; hasta el momento sólo el periodista costarricense Carlos Soto (2016) y la curadora panameña Gladys Turner Bosso (2016) hacen mención a las intervenciones que la conforman. Gladys Turner escribe,

A todo esto hay que sumarle el uso innovador del sitio web de la bienal como espacio alternativo de exposición para artistas cuyos trabajos se centran en la experimentación sonora o audiovisual, y que se constituyó en una verdadera ocasión para salirse de los causes habituales.

De esta manera, la X SONORA, la plataforma que integra herramientas tecnológicas de uso 
cotidiano (Soundcloud, Youtube, etc.), es el espacio donde pueden revisarse trabajos como los del colectivo costarricense Jalamelule, dúo de Djs que experimentan con la mezcla de sonidos electrónicos y ritmos de la música popular latinoamericana; las aportaciones de Mar Alzamora (panameña) quien explora las posibilidades del sonido para evocar y cuestionar los avances del crecimiento urbano descontrolado que arrebata espacios a la convivencia y a la memoria; o el colectivo Uncanny Valley Project (costarricense), creadores con sólida formación musical y artística que proponen la ruptura con viejos modelos estéticos y fórmulas patriarcales (Turner, 2016).

También cabe destacar, en este sentido, la mención que hace Mónica Kupfer al proyecto de Ela Spalding para la sede de la Bienal, que tomó el edificio de la United Fruit Company en el puerto de Limón - Caribe de Costa Rica-, y cito: "La música también fue vehículo expresivo de Ela Spalding para crear un 'puente sonoro de solidaridad' entre las ciudades de Limón y Colón con una rocola que reproducía canciones de calipso populares entre ticos y panameños de origen afrocaribeño" (Kupfer, 2016-2017: 74).

\section{Escuchar desde la proximidad crítica}

Concluir nunca es fácil. Retomando las propuestas de Rubén López Cano y Úrsula San Cristóbal, en la investigación artística debemos aprender a identificar cuándo "cerrar" un proceso (López Cano y San Cristóbal, 2014: 179, 182) y dejar que los rizomas de la intervención se expandan, ajenos a nuestro control científico. Digamos que las quimeras nos enseñan más en estado libre, anárquico. Las creadoras participantes siguieron cada una su rumbo. El espacio de sociabilidad que se creó, una vez cumplido su propósito, se disolvió. Ahora únicamente existe en la redes... se mantuvieron vivas sólo las relaciones entre creadoras que mantenían vínculos afectivos previos. La proximidad es guiada por esas políticas del deseo que menciona Miguel A. López, las cuales trazan, como escribe Tamara Díaz, constelaciones, tejiendo: "[...] líneas imaginarias de un punto a otro, buscando conexiones y vínculos que pudieran ser ampliados y/o cuestionados en cualquier momento" (Díaz, 2003: 11).

Uncanny Valley Project evidenció la necesidad de espacios en los que explorar estas proximidades, sumando el feminismo como pensamiento crítico. Pero la proximidad también tiene sus aporías. En América Latina tenemos la mala costumbre de no apreciar la innovación producida en nuestra propia casa, y eso muy a pesar del pensamiento decolonial. Pareciera que necesitamos saber que ya lo hicieron antes en un lugar "de primer mundo", y que ha sido avalado por instituciones y especialistas "de allá”; sólo así se escucha y valora la innovación que nuestras vecinas nos ofrecen. Consecuentemente, finalizo conectando los resultados de esta intervención ciberfeminista en la X Sonora de la Bienal Centroamericana, con un artículo de Cathy Lane (2016) donde esta autora llama la atención sobre el evento que organizó la Tate Modern en mayo de 2012, titulado: Her Noise: Feminism and the Sonic. ${ }^{52}$

El evento consistió en una serie de actividades cuyo propósito fue investigar los discursos feministas en sonido y música a través de un programa de charlas, presentaciones, debates y proyecciones de películas. Se contó con la presencia de la prestigiosa compositora Pauline Oliveros —fallecida en 2016-, se proyectó el documental The Voice Is a Language dedicado al legado de Meredith Monk, y se realizó un simposio en el que se exploraron y desarrollaron los discursos feministas emergentes en sonido y música, y que reunió a músicas, artistas, académicas y escritoras, para desafiar lecturas estándar acerca de los feminismos y lo sonoro. En el simposio participaron especialistas como Ute Meta Bauer, Fender Schrade, Lina Dzuverovic, Cathy Lane, Salomé Voegelin, Sonia Boyce, Emma Hedditch, Catherine Grant, Georgina Born, Viv Corringham, Anne Karpf, Maggie Nicols, Cara Tolmie, Sue Tompkins, Kaffe Matthews, Nina Power, Tara Rodgers y Anne Hilde Neset. ${ }^{53}$

La intervención ciberfeminista Uncanny Valley Project evidenció cómo los cuerpos de la innovación, y los discursos sobre la creación sonora con nuevos 
medios en la región centroamericana, no constituyen un ámbito de dominio de patriarcas bajo cuyas reglas deben regirse los espacios y gestionarse las tecnologías. Necesitamos, coincidiendo con el evento organizado por la Tate Modern, desafiar lecturas estándar acerca de los feminismos y lo sonoro. Existe una red de creadoras activas, y sus propuestas nos permiten conocer más de cerca la diversidad del saber sonoro de la región centroamericana.

\section{Notas}

${ }^{1}$ TEOR/ética es una de las instituciones de referencia del arte contemporáneo centroamericano. Web oficial: http://teoretica.org/ (consultado el 30 de julio de 2016).

2 La ironía al referirse a San José, capital de Costa Rica, como "país", señala hacia el vallecentralismo dominante en las políticas culturales y socioeconómicas del gobierno e instituciones locales.

3 "Costa Rica toma la X Sonora". En X Bienal Centroamericana, 10 de mayo 2016. Disponible en: http://www.bienalcentroamericana.com/2016/05/10/ costa-rica-toma-la-x-sonora/ (consultado el 29 de julio de 2016).

4 "Noise music is a category of music that is characterised by the expressive use of noise within a musical context. This type of music tends to challenge the distinction that is made in conventional musical practices between musical and non-musical sound" (Priest, 2013: 132).

5 “EP stands for 'extended play record' or simply 'extended play.' An EP is a compilation of songs often created for promotional use and covers the middle ground between a single and a full-length album. EPs are usually around 4 to 6 songs in length and are generally made using original tracks never released before by an artist" (McDonald, 2016).

6 "Science fiction (often shortened to SF, sci-fi or scifi) is a genre of speculative fiction, typically dealing with imaginative concepts such as futuristic science and technology, space travel, time travel, faster than light travel, parallel universes, and extraterrestrial life. Science fiction often explores the potential consequences of scientific and other innovations, and has been called a "literature of ideas" (Gilks, Fleming y Allen, 2003).

${ }^{7}$ Pablo Murillo es el responsable de la imagen de Saturno Devorando, Señorita Abril y Susana López (Bengalas).

8 La autora es una de las creadoras sonoras centroamericanas incluidas en la intervención realizada en la X Sonora de la Bienal Centroamericana (2016).

9 Previo a este proyecto, la única colaboración documentada entre el grupo de Costa Rica y las creadoras centroamericanas es el concierto de la DJ Pasumopi (Honduras) y la banda CoLoRnOiSe (Costa Rica), realizado el 20 de noviembre de 2015 en el Hoxton Pub del Boulevard Los Yoses, San José.

${ }^{10}$ La descripción del grupo "Women in Experimental" indica: "female artists in noise, experimental, ambient, drone, field recordings, free improv, circuit bent, musique concrète, glitch, breakcore, anti-folk..."Web asociada: http://ambientnoise-wall.blogspot.com.es/ Disponible en: https://www. facebook.com/groups/women.experimental.music/ (consultado el 29 de julio de 2016).

${ }^{11}$ El grupo "Mujeres en la experimentación sonora // Latinoamérica" en su descripción indica: "Este es un grupo para que empecemos a conocernos y a estar al tanto del trabajo de mujeres que experimentan con sonido en toda Latinoamérica". Disponible en: https:// www.facebook.com/groups/1622622487999222/ (consultado el 29 de julio de 2016).

${ }^{12} \mathrm{El}$ grupo "FFF" en su descripción indica: "Forum for women composers to discuss issues relevant to our field, exchange ideas and experiences and put our collective heads and talents together to break down barriers and chart our chosen course". Disponible en: https://www. facebook.com/groups/404592236304720/ (consultado el 29 de julio de 2016).

${ }^{13}$ La página "Musicología feminista" está disponible en: https://www.facebook.com/Musicolog\%C3\%ADafeminista-122474581171096/ (consultado el 29 de julio de 2016).

${ }^{14} \mathrm{La}$ página de "MUSERUOLE" está disponible en: https://www.facebook.com/MUSERUOLE-womenin-experimental-music-197860860337561/ (consultado el 29 de julio de 2016). 
15 "Her Noise Archive" actualmente está sostenido por el CRiSAP de la Universidad de las Artes de Londres. Disponible en: http://hernoise.org/ (consultado el 29 de julio de 2016).

16 "Feminatronic. Celebrating the Eclectic Creativity of Women in Electronic Music". Disponible en: http:// feminatronic.com/ (consultado el 29 de julio de 2016).

1 "Female:pressure. international network of female artists in electronic music". Disponible en: www. femalepressure.net (consultado el 29 de julio de 2016).

${ }^{18}$ Programa "Women in Electronic Music", del Canadian Music Centre. Disponible en: http://www.musiccentre.ca/ regions/ontario/projects/women-in-electronic-music?field event_region_value_many_to_one=2 (consultado el 29 de julio de 2016).

${ }^{19}$ Web Oficial de "Femme Cult": http://www.femmecult. com/(consultado el 29 de julio de 2016).

20 "La Biónica" en la página oficial de Festival INTERFASE está disponible en: http://cuerpoytecnologia.cl/la_bionica convocatoria.html (consultado el 29 de julio de 2016).

${ }^{21}$ Página oficial disponible en: http://microcircuitos.org/ (consultado el 29 de julio de 2016).

22 Costa Rica en la plataforma "Microcircuitos", disponible en: http://costarica.microcircuitos.org/ (consultado el 29 de julio de 2016).

23 La página de DEBATES SONOROS-UCR está disponible en: https://www.facebook.com/debatesonoro (consultado el 29 de julio de 2016).

${ }^{24}$ Una descripción acerca del CES-UCR está disponible en: http://artesmusicales.ucr.ac.cr/investigacion/ces/ (consultado el 29 de julio de 2016).

25 Otto Castro hace referencia a Mariela Richmond y Paulina Velásquez, ya que ambas fueron pioneras al participar representando a Costa Rica en el Forma $\&$ Sustancia. Festival Internacional de Performace, celebrado en la Alianza Francesa de Guatemala en 2013. Disponible en: https:/multifungi.com/2013/03/20/ mv-festival-internacional-de-performance-guatemala/ (consultado el 29 de julio de 2016).

${ }^{26}$ Para una actualización del estado de la cuestión ver Cascudo y Aguilar-Rancel (2013).

27 Favorecida por trabajos como Feminismo y música. Introducción crítica (Narcea, 2003) de Pilar Ramos. La propia autora reflexiona acerca de este impacto en su artículo de 2010, "Luces y sombras en los estudios sobre las mujeres y la música”.

28 Programa disponible en: https:/issuu.com/ cicomucr/docs/programa_de_encuentro_feminismos v2_2e3d99bee2cdb9 (consultado el 29 de julio de 2016).

${ }^{29}$ Programadisponibleen:http://costarica.microcircuitos. org/i-encuentro-sobre-estudios-sonoros-y-creacionexperimental/ (consultado el 29 de julio de 2016).

30 Web oficial de Susan Campos Fonseca: www. susancamposfonseca.com (consultado el 29 de julio de 2016).

${ }^{31}$ José Duarte. Comentario publicado en Facebook con fecha del 20 de mayo de 2016.

32 "Time" (Videoclip, 2013) de CoLoRnOiSe. Disponible en: https://www.youtube.com/watch?v=A0zAcCvkM Sg\&\&feature $=$ share\& list $=$ PLBRLtnDp7CACoeA_8Rii24 fES3AhbTqyp\& \&index $=6$ (consultado el 29 de julio de 2016).

33 Campos Fonseca, Susan. Comentario publicado en Facebook el 19 de mayo de 2016.

${ }^{34}$ Convocatoria publicada en: http://medialab-prado. es/article/editatona-sobre-compositoras-de-musicaelectronica-en-wikipedia-9j (consultado el 29 de julio de 2016).

35 Playlist publicada en: https://playmoss.com/en/elestado-mental/playlist/early-sheynth (consultado el 29 de julio de 2016).

36 Video publicado en: https:/www.facebook.com/ PlayGroundMag/videos/1153367534703121/ (consultado el 29 de julio de 2016).

${ }^{37}$ Web oficial Women in Sound Women on Sound: http://wiswos.bitbucket.org/index.html?p=435.html (consultado el 29 de julio de 2016).

${ }^{38}$ SHE MAKES NOISE coordinado por Natalia Piñuel: http://shemakesnoise.tumblr.com/ (consultado el 29 de julio de 2016).

${ }^{39}$ Recuérdese que era la primera vez que se planteaba una intervención sonora en términos ciberfeministas en la región, esto a pesar de que investigadoras como Zamira Barquero, Tania Vicente, Tania Camacho, Karla Abarca y la autora vienen desarrollando estudios sobre mujeres en la música desde aproximadamente 2008. 
${ }^{40}$ Proyecto 01-158-2016 “Abordajes de la experimentación y aplicación de nuevas tecnologías sostenibles en la creación sonora", inscrito en la Sede del Atlántico (Recinto Turrialba) de la UCR. Investigadora responsable: Dra. Susan Campos Fonseca.

${ }^{41}$ En el caso particular de esta autora, musicóloga y compositora, era una oportunidad de colaborar con creadoras alasquehabía venidoestudiando, en el marcode un proyecto dedicado a la experimentación y utilización de nuevas tecnologías en la creación sonora producida en Costa Rica. Esta intervención venía precedida por un proceso, cuyos resultados se habían materializado en: “iArqueologías sonoras del presente?", conferencia dictada en el Congreso Internacional de Musicología de Casa de las Américas, desarrollado en La Habana en 2014; "Noise, Sonic Experimentation and Interior Coloniality in Costa Rica", ponencia en el Simposio Experimental Music in Practice: Prespectives from Latin America, desarrollado en Rutgers, The State University of New Jersey, en 2015; y "Ciberfeminismo y estudios sonoros", comunicación presentadaen el IIICongreso Internacional Espacios sonoros y audiovisuales. "La ciudad como lugar de experimentación, creación e investigación plurisensorial", desarrollado en la Universidad Autónoma de Madrid en 2016. Debo informar sobre estos antecedentes porque la intervención en la X Sonora de la Bienal era parte de un tejido académico que venía hilando desde 2013.

42 Sonora Desconferencia, celebrada en el marco del II Encuentro de Estudios Sonoros y Creación Experimental. Programa disponible en: http://costarica.microcircuitos. org/ii-encuentro-sobre-estudios-sonoros-y-creacionexperimental/ (consultado el 23 de diciembre de 2016).

${ }^{43}$ Programa completo del I Encuentro disponible en: http:// costarica.microcircuitos.org/i-encuentro-sobre-estudiossonoros-y-creacion-experimental/ (consultado el 26 de diciembre de 2016).

${ }^{44}$ Uncanny Valley Project Costa Rica, disponible en: https:// soundcloud.com/labienal_ca/sets/uncanny-valleyproject (consultado el 30 de julio de 2016).

${ }^{45}$ Uncanny Valley ProjectCentroamérica, disponibleen: https:// soundcloud.com/labienal_ca/sets/uncannyvalleyproject (consultado el 30 de julio de 2016).
${ }^{46}$ Uncanny by Fiamma Aleotti y Uncanny by Susan Campos, disponibles en: https://open.spotify.com/user/labienal_ ca (consultado el 30 de julio de 2016) .

${ }^{47}$ Canal de la X Bienal Centroamericana disponible en: https://www.youtube.com/playlist?list=PLBRLtnDp 7CACoeA_8Rii24fES3AhbTqyp (consultado el 23 de diciembre de 2016).

48 La memoria fotográfica del Uncanny Valley Project está disponible en: www.facebook.com/ susancamposcomposer (consultado el 30 de julio de 2016).

${ }^{49}$ Debe informarse que la cantante Sofie Letitre sacó al mercado en 2015 un álbum titulado Uncanny Valley Project. También se celebró el "Brooklyn Electronic Music Festival: Freaking Fawning over Sinjin Hawke, Zora Jones and Martyn Bootyspoon's Fractal Fantasy 'Uncanny Valley' project. Prepare for an audio-visual facemelt" (6 de octubre de 2015).

${ }^{50}$ Lease cita original completa: 15 de mayo, 21.00 Italian time-UTC/GMT+1, UNEXPLAINED SOUNDS GROUP web radio transmission, The Recognition Test \# 20, curated by Sonologyst. Streaming: http://mixlr.com/ unexplained-sounds/ (consultado el 30 de julio de 2016).

${ }^{51}$ Difference - visions of contemporary composition (2017). Álbum disponible en: https://unexplainedsoundsgroup. bandcamp.com/album/differences-visions-ofcontemporary-composition (consultado el 10 de enero de 2017).

${ }^{52}$ Her Noise: Feminism and the Sonic (Tate Modern, mayo de 2012), programa disponible en: http://www.tate.org. uk/whats-on/tate-modern/her-noise-feminisms-andsonic (consultado el 30 de julio de 2016).

${ }^{53}$ Los audios del simposio están disponibles en: http://www. tate.org.uk/context-comment/audio/her-noise-symposiumaudio-recordings (consultado el 30 de julio de 2016).

\section{Referencias bibliográficas}

Allan, Jennifer Lucy (2016). "Mothers of invention: the women whopioneered electronic music". En The Guardian, 17, junio. Disponible en: https://www.theguardian.com/ music/2016/jun/17/daphne-oram-synthesizer-deepminimalism (consultado el 29 de julio de 2016). 
Born, Georgina y David Hesmondhalgh (2000). Western Music and Its Others. Difference, Representation, and Appropriation in Music. Berkeley: University of California Press.

Born, Georgina (1993). "Afterword: Music Policy, Aesthetic and Social Difference". En Tony Bennett et al. (ed.), Rock and Popular Music: Politics, Policies, Institutions. Nueva York: Routledge, pp. 266-292.

Burden, Zora von (ed.) (2010). Women of the Underground: Music: Cultural Innovators Speak for Themselves. San Francisco, CA: Manic D Press.

Campos Fonseca, Susan (2016a). "Ciberfeminismo y estudios sonoros". En INTERdisciplinaria. Revista del Centro de Investigaciones interdisciplinares en Ciencias y Humanidades, 4(8): 151-158, enero-abril. Ciudad de México: Universidad Nacional Autónoma de México.

Campos Fonseca, Susan (2016b). "Cyberfeminismo y estudios sonoros: una intervención en X SONORA de la Bienal Centroamericana 2016". Conferencia dictada el 8 de julio de 2016 en el Centro de Estudios Superiores de México y Centroamérica de la Universidad de Ciencias y Artes de Chiapas (CESMECA-UNICACH). San Cristóbal de las Casas, Chiapas, México. Disponible en: http://www.cesmeca.mx/index.php/jopbail/122boletin0123 (consultado el 30 de julio de 2016).

Campos Fonseca, Susan (2014). “iArqueologías sonoras del presente?". En Boletín Música. Estudios Culturas Originarias de América, 37: 27-43. La Habana: Casa de las Américas.

Campos Fonseca, Susan e Isabel Porto Nogueira (eds.) (2013). Estudos de gênero, corpo e música: abordagens metodológicas. Goiânia, Brasil: ANPPOM.

Campos Fonseca, Susan y Josemi Lorenzo Arribas (eds.) (2011). "Música y estudios sobre las mujeres/Music and Women's Studies". En Transcultural,15. Disponible en: http://www.sibetrans.com/trans/publicacion/16/ trans-15-2011 (consultado el 29 de julio de 2016).

Campos Fonseca, Susan (en prensa). "Noise, Sonic Experimentation and Internal Coloniality".

Cascudo, Teresa y Miguel Ángel Aguilar-Rancel (2013). "Género, musicología histórica y el elefante en la habitación”. En Susan Campos Fonseca e Isabel Porto Nogueira (eds.), Estudos de gênero, corpo e música: abordagens metodológicas. Goiânia, Brasil: ANPPOM, pp. 27-55.

Chacón, Vinicio (2013). “iArtistas sonoros uníos!". En Semanario Universidad, 31 de julio. San José, Costa Rica: Universidad de Costa Rica. Disponible en: http://semanariouniversidad.ucr.cr/cultura/artistassonoros-unos/ (consultado el 29 de julio de 2016).

Chávez-Espinach, Fernando (2016). "Susan Campos: inquieta creadora de sonidos". En La Nación, 25 de mayo. Disponible en: http:/www.nacion.com/ ocio/musica/Susan-Campos-inquieta-creadorasonidos_0_1562843740.html (consultado el 30 de julio de 2016).

Creed, Ryan (2016). "18 Women In Electronic Music You Need To Hear Right Now". En BuzzFeed, 18 de abril. Disponible en: https://www.buzzfeed.com/ ryancreed/women-in-electronic-music-you-need-tohear-right-now?utm_term=.hdAdYa2j6\#.rmnvbJZlq (consultado el 29 de julio de 2016).

Díaz, Tamara (2016a). Crítica próxima : Critical proximity. San José, Costa Rica: TEOR/ética.

Díaz, Tamara (2016b). Todas las vidas. Disponible en: http://www.bienalcentroamericana.com/todas-lasvidas/ (consultado el 29 de julio de 2016).

Díaz, Tamara (2003). En el trazo de las constelaciones. San José, Costa Rica: Ediciones Perro Azul.

Gilks, Marg, Paula Fleming y Moira Allen (2003). "Science Fiction: The Literature of Ideas". En Writing-World.com. Disponible en: http://www. writing-world.com/sf/sf.shtml (consultado el 15 de marzo de 2017).

Haraway, Donna Jeanne (1995). Ciencia, cyborgs y mujeres: la reinvención de la naturaleza. Valencia: Universitat de València.

Kageki, Norri (2012). “An Uncanny Mind: Masahiro Mori on the Uncanny Valley and Beyond”. En IEEERoboticse Automation Magazine, 19(2), junio. Disponible en: http:// spectrum.ieee.org/automaton/robotics/humanoids/ an-uncanny-mind-masahiro-mori-on-the-uncannyvalley/ (consultado el 23 de diciembre de 2016).

Kupfer, Mónica (2016-2017). "Arte y autorreflexión: Décima Bienal Centroamericana”. En Art Nexus, 103, diciembre 2016-febrero 2017. 
Lane, Cathy (2016). "Sound::Gender::Feminism::Activi sm: Research and Challenges to the Orthodoxies of Sound Arts". En Contemporary Music Review, julio, 35(1): 32-39.

López, Miguel A. (ed.,) (2016). Crítica próxima: Critical proximity. Tamara Díaz (aut.). San José, Costa Rica: TEOR/ética.

López Cano, R. y Úrsula San Cristóbal Opazo (2014). Investigación artística en música. Problemas, experiencias y modelos. Barcelona: Fonca, EsMuC.

McDonald, Heather (2016). "Music Marketing: What Is an EP? How to Get Started Creating Your Own Extended Play Record". En The Balance, 22 de abril. Disponible en: https://www.thebalance.com/musicmarketing-what-is-an-ep-2460346 (consultado el 15 de marzo de 2017).

McClary, Susan (2011). "Feminine Endings at Twenty". En Transcultural, 15. Disponible en: http://www.sibetrans. com/trans/public/docs/trans_15_02_McClary.pdf (consultado el 29 de julio de 2016).

McClary, Susan (1991). Feminine Endings. Music, Gender, and Sexuality. Minneapolis, MN: University of Minnesota Press.

Mori, Masahiro, Karl F. MacDorman y Norri Kageki (2012). "The Uncanny Valley [From the Field]". En IEEERobotics $\checkmark$ Automation Magazine, 19(2), junio. Disponible en:http:// ieeexplore.ieee.org/document/6213238/ (consultado el 22 de diciembre de 2016).

Priest, Eldritch (2013). "Music Noise" in Boring Formless Nonsense: Experimental Music and The Aesthetics of Failure. Londres y Nueva York: Bloomsbury Publishing, Bloomsbury Academic.

Ramos, Pilar (2003). Feminismo y música. Introducción crítica. Madrid: Narcea.

Ramos, Pilar (2010). "Luces y sombras en los estudios sobre las mujeres y la música“. En Revista Musical Chilena, 64(213): 7-25, enero-junio.
Richter, Katrin(2013)."Women and Their Machines: A Think-piece about Female Pioneerism in Electronic Music, Post-post Feminism and Some Sassy Statements on Sexism". EnMeokonet,18 deabril. Disponibleen: http:// www.meoko.net/news/women-and-their-machinesa-meoko-think-piece-about-female-pioneerism-inelectronic-music (consultado el 29 de julio de 2016).

Rodgers, Tara (2010). Pink Noises: Women on Electronic Music and Sound. Durham, NC: Duke University Press. Santamaría, Alberto (2016). Paradojas de lo Cool. Arte, Literatura y Política. Santander, España: La Vorágine.

Soto Campos, Carlos (2016). "Sonidos experimentales tendrán su casa en la Universidad de Costa Rica”. En La Nación, 24 de mayo. Disponible en: http://www. nacion.com/ocio/musica/Sonidos-experimentalesUniversidad-Costa-Rica_0_1562643768.html (consultado el 30 de julio de 2016).

Spíndola Zago, Octavio (2016). "Espacio, territorio y territorialidad: una aproximación teórica a la frontera". En Revista Mexicana de Ciencias Políticas y Sociales, Nueva Época, LXI(228), septiembre-diciembre. México: Universidad Nacional Autónoma de México.

Turner Bosso, Gladys (2016). “Todas las vidas importan en la X Bienal Centroamericana”. En Domus, diciembre.

VV.AA. "Introducción" (1994). En Perspectives of New Music, invierno, 32(1): 6-7.

Weiner, Sophie (2016). "DJ Maria Chavez Embraces Beautiful Destruction on the Turntable". En The Village Voice, 5 de mayo. Disponible en: http://www. villagevoice.com/music/dj-maria-chavez-embracesbeautiful-destruction-on-the-turntabVle-8579191 (consultado el 29 de julio de 2016).

\section{Sitios web de referencia}

http://www.bienalcentroamericana.com/ http://www.susancamposfonseca.com/ 
Foto. 1. Señorita Abril

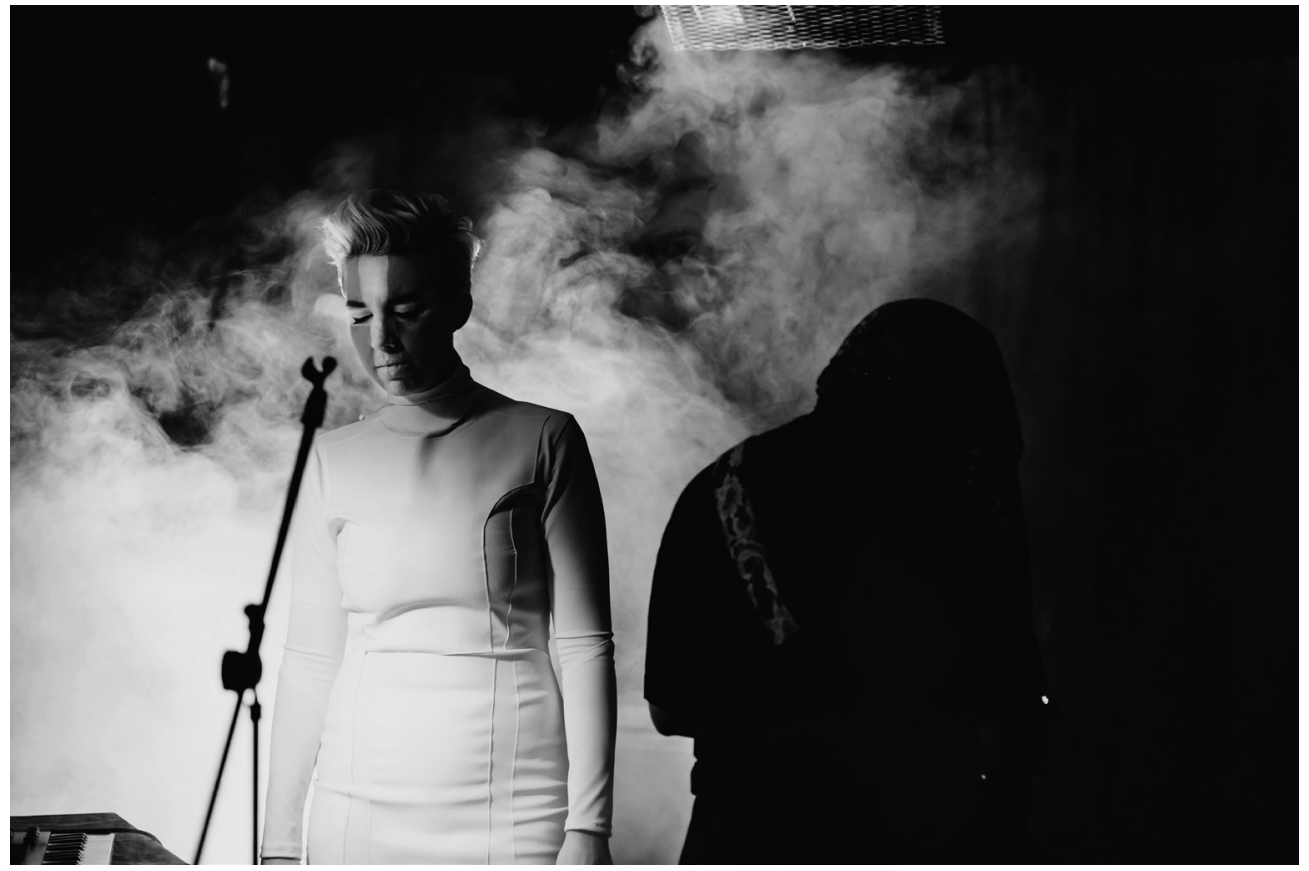

Fuente: Fotografía de Pablo Murillo, 2015.

\section{Foto 2. Saturno Devorando}

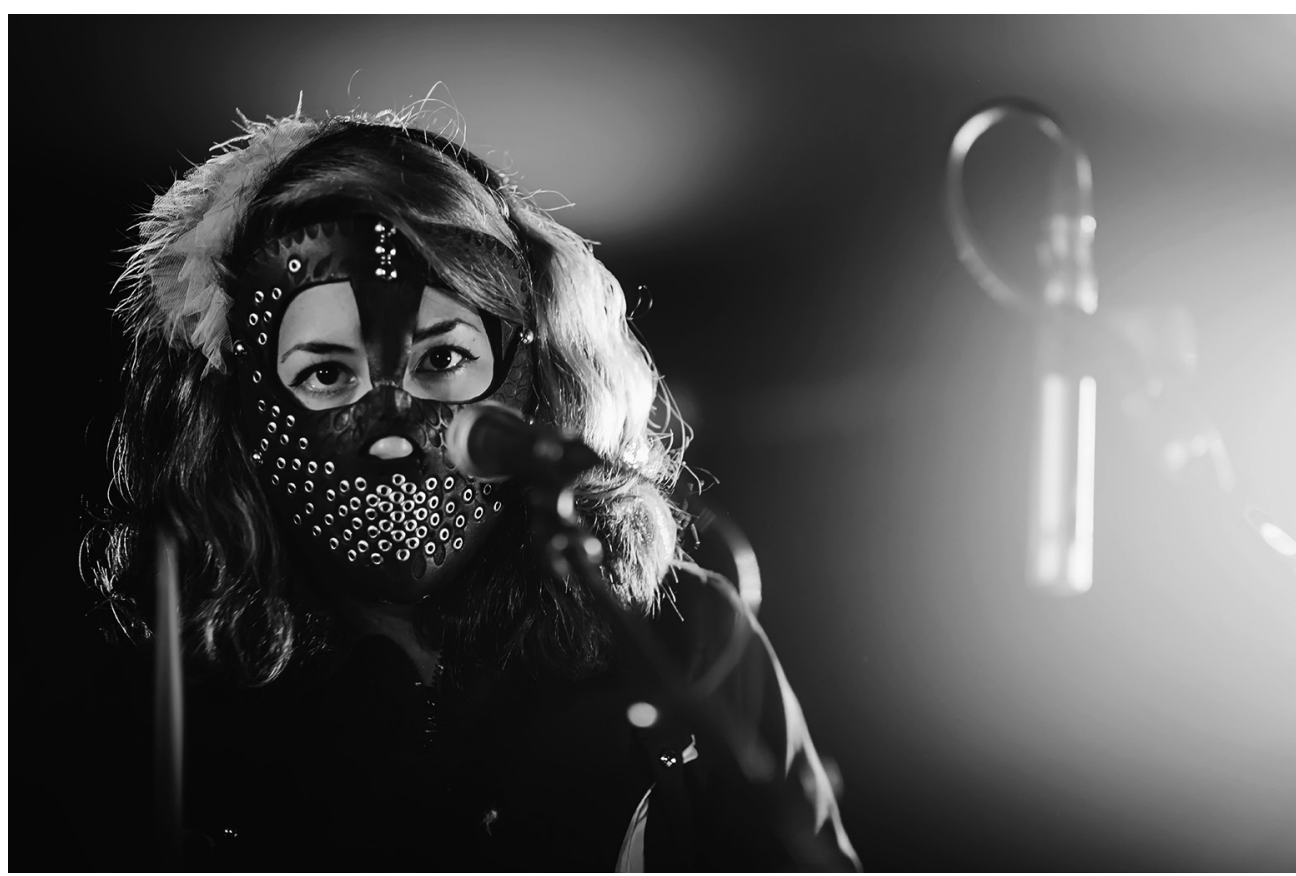

Fuente: Fotografía de Pablo Murillo, 2015. 
Foto 3. "La Inmaculada Conceptual" de la serie Métodos Anticonceptuales

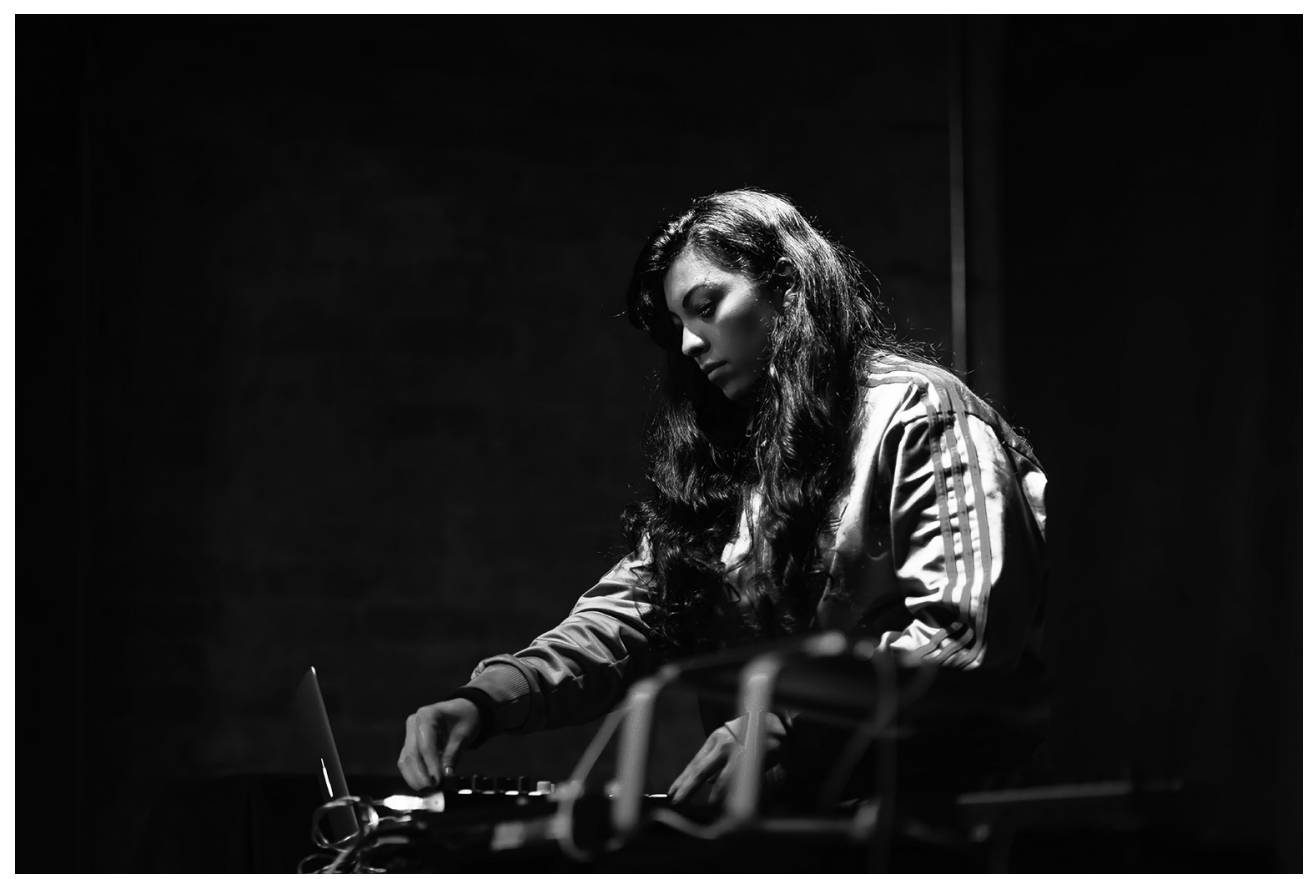

Fuente: Fotografía de Pablo Murillo, 2015.

Foto 4. DJ Monik Zdan
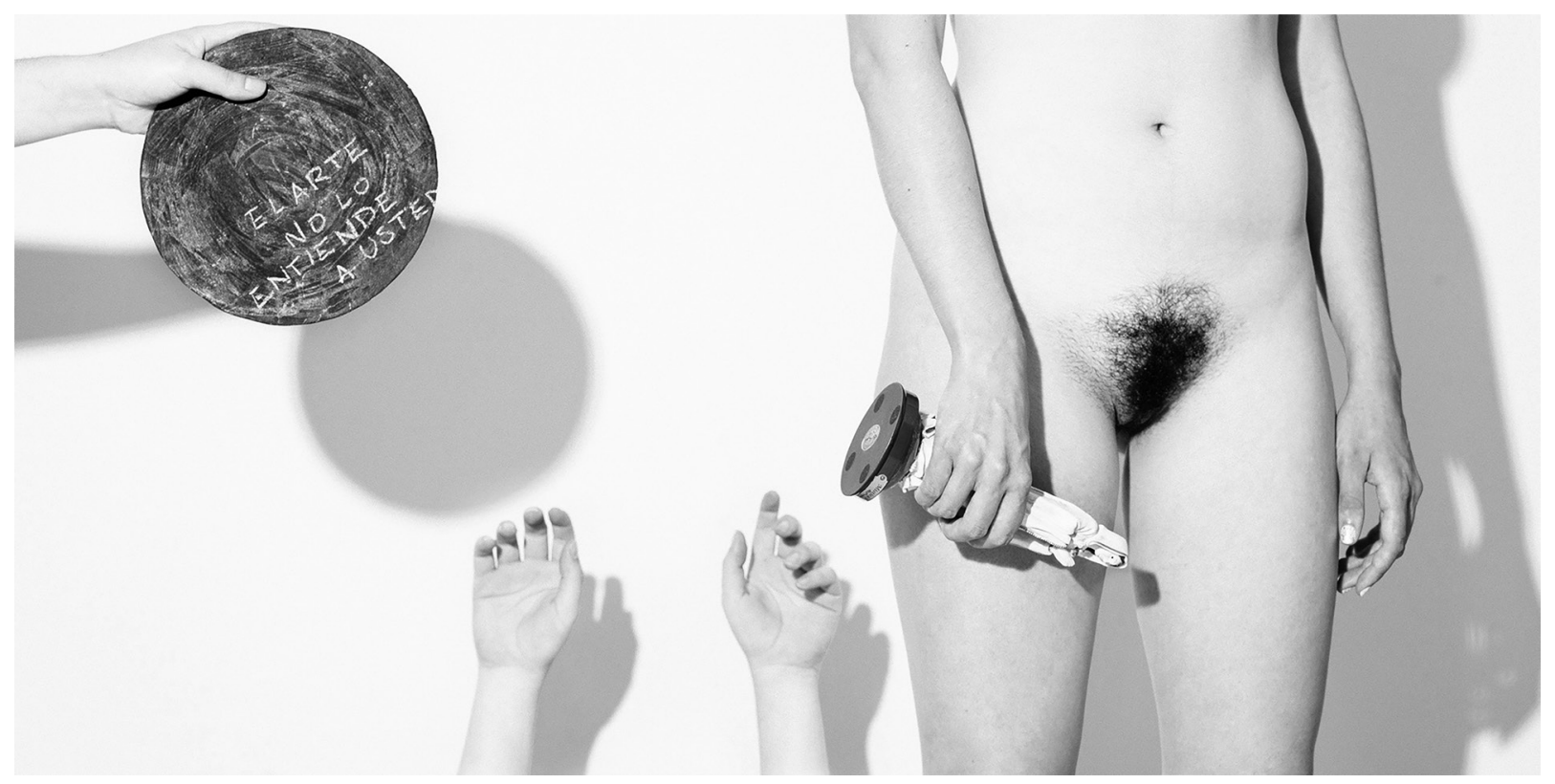

Fuente: Fotografía de Pablo Murillo, 2016. 